\title{
WIRELESS CLOUD ARCHITECTURE BASED ON THIN CLIENTS AND ONTOLOGIES
}

\author{
Todor Cooklev ${ }^{1}$, Lubomir Stanchev ${ }^{2}$, Chao $\mathrm{Chen}^{3}$ \\ Indiana University Purdue University Fort Wayne \\ 2101 E Coliseum Blvd., Fort Wayne, Ind., 46805, USA \\ cooklevtaipfw. edu \\ stanchel@ipfw.edu \\ chencaipfw.edu
}

\begin{abstract}
Recently, several researchers have discovered the need for radios to use description techniques for the objects in the wireless realm. The concept of RF field-programmable analog array (FPAA) was also proposed recently and the lack of hardware abstractions was identified as a problem. We propose a hardware abstraction for RF FPAAs, which enables an open RF-digital interface. We advance the concept of wireless thin clients. These clients are connected to the cloud using the open RF-digital interface. We describe the architecture of a comprehensive wireless ontology.
\end{abstract}

\section{INTRODUCTION}

Modern civilian, public-safety, and military wireless networks are highly heterogeneous. It is highly desirable for new radios, services, and applications to be readily incorporated, without significant changes elsewhere in the network. One of the prerequisites for achieving this goal is the development of a language that can be used to describe both the capabilities of RF components and their current operational status. In traditional radio design, description techniques are not necessary because all the metadata is constant and it therefore can be assumed known. The need for such a language is recognized in several publications [16]. Here we extend these previous works and in Section 2 we propose a hardware abstraction for RF fieldprogrammable analog arrays (FPAAs). Furthermore, we extend the cloud concept to the radio world and in Section 3 propose a radio architecture consisting of thin radio clients connected over a packet-based interface to a wireless cloud. Section 4 is devoted to a comprehensive cognitive radio ontology that is the foundation of semantic wireless networking.

\section{HARDWARE ABSTRACTION FOR RF FPAAs}

RF field-programmable analog arrays (FPAAs) are reconfigurable integrated circuits for analog RF signal processing. Not only their components are software-defined, but their topology is also software-defined. Here we consider a "coarse-grain" reprogrammable RF FPAA that consists of multiple amplifiers, mixers, filters, matching networks, etc. RF FPAAs are attractive because they can implement radio access technologies (RATs) to be defined in the future, i.e. they are "future-proof" [1]. Currently RF ICs are optimized for a single RAT and are re-designed for every new RAT.

The need for an appropriate Hardware Description Language (HDL) is known [1]. To develop an HDL for RF mixed-signal programmable ICs, we extend the VITA-49 standard [7]. VITA-49 defines an open interface for a radio receiver with a fixed topology [7]. It defines a packet-based interface, i.e., it is a software bus. There are packet encoders and parsers/decoders on either side of the connection. We extend VITA 49 and define control and extension control packets, in addition to the data and context packets. The meaning of the context packets is also extended and they include parameters that pertain to both the transmitter and receiver such as those defined in [7], plus additional parameters such as reconfiguration time, partial vs. full reconfiguration. Collectively these parameters describe completely the RF FPAA. Every time one or more of these parameters change, the RF encoder sends a context packet containing the current value of the parameters that have changed. Initially, the RF IC sends an extension context packet to describe its possibilities. The topology (and therefore the performance) can be reconfigured using commands in a way that is similar to the reconfiguration process of a digital gate-level FPGA. The control packets are generated on the digital hardware side and contain metadata also pertaining to both the transmitter and receiver.

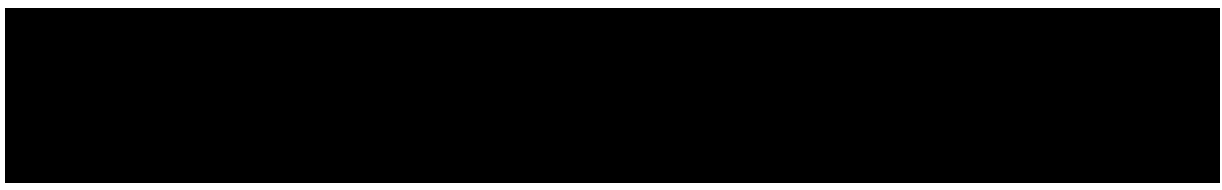


These control packets are the reconfiguration commands. Controlling the topology of a coarse-grain RF FPAA is done using reference points. The control packet decoder translates the metadata to the hardware settings of each component on the RF FPAA. Note that this description has a hierarchical structure, because the metadata descriptions of several circuit elements can be combined into aggregate context or control packets. This aggregate control or context packet describes the entire RF FPAA.

\section{WIRELESS THIN CLIENTS AND CLOUDS}

The area of cloud computing has experienced significant growth recently and there have been attempts to transfer the cloud concept to wireless systems [8-12]. In most cases "wireless cloud" means a collection of radios or a collection of base stations connected using the X2 interface. In these cases the connection to the cloud is over the air interface and the "client" is a stand-alone (or fat) radio. We propose a different type of wireless architecture consisting of a thin client and a cloud, connected using a packet-based interface, illustrated in Figure 1. A radio thin client can be loosely defined as a radio without the capability to perform modulation and coding. This thin radio client includes, in general, a software-defined front-end such as an RF FPAAs, some mixed-signal elements such as data converters, and even some digital hardware. The digital hardware implements digital signal processing that is independent of the radio access technology (RAT) such as decimation/interpolation. The thin client may also include digital signal processing hardware to perform spectrum sensing. The digital signal processing (modulation, coding, etc.) and all higher-layer protocols that implements the RAT take place in the cloud. (The cloud concept blurs to some extent the distinction between radio and $R F$ device.) The thin radio cannot decode signals on its own, but can act as an amplify-and-forward relay. The cloud provider manages the infrastructure that runs the radio software. In the basic model clouds can offer infrastructure as a service (IaaS), where the cloud offers only digital hardware. There are higher levels of service such as "platform as a service", where in addition to digital hardware, the cloud offers system-level software, and "radio software as a service", where the software that implements a RAT together with the cognitive engine (CE) is offered as a service. In this model users are given access to radio software on an "on-demand" basis. This eliminates the need to install and run the radio software on the radio platform, which simplifies maintenance and support.

There are some key differences between radio clouds and computing clouds. One difference is the interface between the thin client and the cloud. In computing clouds this interface is trivial and is usually TCP/IP. In radios the interface is not a trivial issue. To be able to attach seamlessly thin clients to the cloud the RF-digital interface must be open and software-based; none of the closed interfaces $[13,14]$ that currently exist are appropriate.

Another difference between computing and radio clouds is that thin computing clients cannot offer any services to the cloud, while thin radio clients can offer certain services (such as spectrum-sensing) to the cloud.

The cloud concept allows significant new opportunities, particularly for cognitive radios. The CE is an "intelligent" agent that manages the cognition tasks in a cognitive radio. The $\mathrm{CE}$ can be implemented as an independent entity interacting with the reconfigurable RF front-end, or as a collection of interacting entities each fulfilling a specific role. One of the important tasks for the $\mathrm{CE}$ is to determine which radio protocol is active at any one time, and at what parameters, such as RF center frequency and RF power this protocol will operate. Cognitive devices should dynamically detect available RATs and available resources. Therefore the CE must have domain knowledge of radio communication. Based on this knowledge, the $\mathrm{CE}$ can optimize the various parameters and protocols. There is one important difference between the $\mathrm{CE}$ and other applications, such as web browsing; the CE must have hardware-specific knowledge. Some metadata parameters, such as RF center frequency and RF power level are physically determined only by the RF front-end. On the other hand, in a cloud architecture the CE must be isolated from the underlying hardware. This is possible only with the developed hardware abstraction.

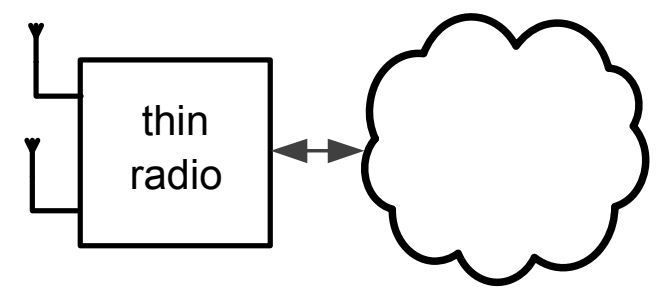

Figure 1 Thin radio client connected to a cloud

\section{COGNITIVE RADIO ONTOLOGY}

Ontology is a general mechanism to describe objects in a certain domain and the relationships among these objects. The Resource Description Framework (RDF) is a simple ontology language that describes things using triplets, e.g., subject, predicate, object. In [3] the Network Description Language (NDL), an RDF-based ontology was proposed. Another example is the Web Ontology Language (OWL) [15]. An OWL-based cognitive radio ontology was proposed in [4]. The Rule Interchange Format (RIF) is another possibility [16]. We envision a comprehensive cognitive radio ontology, including:

1) The current and potential parameters and topology of the RF FPGA. All parameters in the control and context packets should be described. For example, 
Device and

RFFreuqency some $\mathrm{MHz}[>=5000<=6000]$ and

RFBandwidth some $\mathrm{MHz}[>=1<=20]$ and

OutputPower some $\mathrm{dBm}[>=-4<=30]$

The describes a device with RF frequency between 5000 and $6000 \mathrm{MHz}, \mathrm{RF}$ bandwidth between 1 and $20 \mathrm{MHz}$ and output power between -4 and $30 \mathrm{dBm}$. Note that measurement units are not part of OWL. However, reasoning with them can be easily introduced. An example describing the topology of an RF front-end would be as follows.

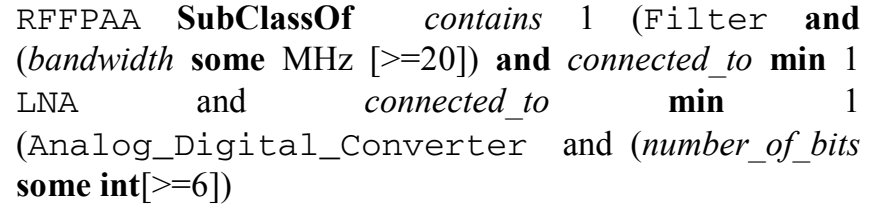

Note that the mapping between such ontology descriptions and the context and control packets introduced earlier is one-to-one. The ontology descriptions are used within the cloud and the control and context packets are used over the interface to the thin radio client.

2) The parameters of the digital hardware. The parameters that need to be described depend on the technology. For example, for FPGAs the parameters are number of configurable logic blocks, number of I/O pins, I/O logic level, configuration method, and power consumption.

3) RATs such as: GSM, Bluetooth, IEEE $802.11 \mathrm{a} / \mathrm{b} / \mathrm{g} / \mathrm{n}$, LTE, including MAC-level protocols such as IEEE802.11r, IEEE802.21, etc.

4) System parameters. Location is the most important system parameter, but there are other parameters that could be useful. For example, battery life can be used in energy-aware computing.

5) Information and user types (types of data, types of users, priorities, Quality of Service (QoS))

6) Current network topology, available networks, and their parameters (data rates, cost of access, security protocols, etc.). For example, to describe a Wi-Fi device that is connected to an Access Point (AP), one can use

Device and connectedTo some (BSSID value [XXXXXX])

The AP with the above BSSID can be described as having Ethernet interface. Note that the device can be connected to other devices as well.

7) Spectrum etiquette parameters. The etiquette includes RF frequency, bandwidth, start time and duration of transmissions, power mask, antenna pattern and polarization, channel monitoring time, channel monitoring bandwidth, etc.

8) Policies, such as regulatory policy, service provider policy, user policy, mission policy, security policy, vendor policy, etc. To resolve potential conflicts among them, policies can have different priorities.

This cognitive radio ontology is broader than [4] in all categories. It also allows new parameters to be easily introduced, since there cannot be one set of parameters acceptable to the entire RF community. Note that the descriptions must be made by multiple domain experts.

\section{SEMANTIC WIRELESS NETWORKS}

Radios that understand the wireless realm form semantic wireless networks. In a semantic wireless network, radio clients can use services that are dynamically discovered without prior negotiations between client and service providers. Note that in addition to radio clients and service providers there may be middle agents; the term "agent" includes all of these. All agents can access and interpret Web-published ontologies, and communicate using messages whose content is represented or can be interpreted, in terms of published ontologies. Automatic service discovery is the automatic location discovery of devices that provide particular services. Currently this service is provided by a human who has to locate the RF device and execute the service manually. Servers can advertise in a service registry or be discovered using an ontology search engine. Service providers publish descriptions of their capabilities using a formal description language, such as OWL. There is a service profile - what inputs does it require and what outputs does it provide, and a service model - how does it work. The ontology must provide declarative API for the automatic execution of the services. Clients can search using an ontology query language, interpret these descriptions, and select appropriate services. As a result of this style of interaction, clients will be able to adjust smoothly when new devices and new RATs are introduced. A semantic wireless network is a highly intelligent network where the radios can, on their own and without human intervention, carry out tasks such as identifying available spectrum and spectrum usage policy; if necessary, buy or rent spectrum; identify available networks, RATs, and decide which one to use, etc. The radios can ensure that the selected RF frequency, RAT, network, etc. will comply with all applicable policies based on geographic location, user preferences, etc. Semantic wireless networks offer "personalization", where different users have access to different parts of the ontology descriptions and the network behaves differently for different clients.

Note that the CE must have a semantic search engine. Previous works such as [6] assume that cognitive radios will have a stand-alone search engine. In a stand-alone search 
engine the crawler browses the description documents of policies, hardware, etc. Then the document metadata is stored in an index based on which the query engine evaluates query requests. The cloud architecture facilitates the semantic search done by the $\mathrm{CE}$ and allows a different type of a search engine, where there is no index of documents. Instead, the search engine distributes queries to other search engines and combines the results afterwards.

Consider the following simple example, where the current regulatory policy specifies three unlicensed bands, ISM1 between 900-928 MHz, ISM 2 between 2400-2483.5 $\mathrm{MHz}$, and UNII bands between 5 and $6 \mathrm{GHz}$. They can be described using the Manchester OWL syntax as follows:

ISM1 SubClassOf Band and Available and (bandwidth some $\mathrm{MHz}[>=900,<=928]$ ) and (maxPower some $\mathrm{mW}$ $[<=1000])$

ISM2 SubClassOf Band and Available (bandwidth some $\mathrm{MHz}[>=2400,<=2483.5])$

and (maxPower some $\mathrm{mW}[<=1000]$ )

UNIILow SubClassOf Band and Available and (bandwidth some GHz $[>=5.15,<=5.25]$ ) and (maxPower some $\mathrm{mW}[<=50]$ ) and (use only Indoor)

UNIIMid SubClassOf Band and Available and (bandwidth some GHz $[>=5.25,<=5.35]$ ) and (maxPower some $\mathrm{mW}[<=250]$ ) and (use only (Indoor or Outdoor))

UNII2 SubClassOf Band and Available and (bandwidth some $\mathrm{GHz}[>=5.47,<=5.725]$ ) and (maxPower some $\mathrm{mW}$ $[<=250]$ ) and (use only (Indoor or Outdoor))

UNIIHigh SubClassOf Band and Available and (bandwidth some GHz $[>=5.725,<=5.825]$ ) and (maxPower someW $[<=1]$ ) and (use only Indoor)

So if the CE makes a query about unlicensed bands, there will be multiple answers. Additional queries or reasoning can be invoked to try to improve the decision. Inference is the process of deducing new information [17]. If the location of the RF device can be inferred, then the choices will be narrowed. Similarly, the CE can infer other parameters such as QoS, user preferences, etc.

\section{CONCLUSIONS}

In this paper by leveraging the VITA-49 standard we introduced a Hardware Description Language for RF FPAAs. We also advance a radio architecture based on thin radio clients connected to a cloud using a packet-based interface. These cognitive radio clouds rely on sharing of resources to achieve economies of scale, and take the concept of converged infrastructure to a higher level. Because it becomes much easier to add to the cloud another thin client, new business models become possible. For example, it becomes possible to have free voice and/or data services with limited QoS or data rate, with revenue derived from the enhanced QoS or from advertising. We describe a comprehensive ontology as the foundation of semantic wireless networks. The ontology will be dynamic and will expand as new radio architectures and solutions are being developed.

\section{REFERENCES}

[1] Radio Frequency-Field Programmable Gate Arrays (RF-FPGA), Microsystems Technology Office, DARPA-BAA-12-13, Nov. 2011.

[2] B. Fette (Ed.), "Cognitive radio technology," Newnes Publication, August 2006.

[3] T. Cooklev, M. Cummings, "Networking Description Language for ubiquitous cognitive networking," SDR Forum Tech. Conf., Washington, DC, 2008.

[4] Wireless Innovation Forum, "Description of the Cognitive Radio Ontology," WINN-10-S-007, Sept. 2010.

[5] L. Stanchev, T. Cooklev, D. Clendenen, "Describing radio hardware and software using OWL-DL for software download and certification," SDR Forum Tech. Conf., Washington, DC, Dec. 2010.

[6] IEEE Std 1900.5 $5^{\text {TM}}$-2011, Policy Language Requirements and System Architectures for Dynamic Spectrum Access Systems

[7] T. Cooklev, R. Normoyle, and D. Clendenen, "The VITA 49 RF-digital interface," IEEE Circuits Systems Magazine, Dec. 2012.

[8] M. Webb, Li Zhaojun, P. Bucknell, T. Moulsley, S. Vadgama, "Future Evolution in Wireless Network Architectures: Towards a 'Cloud of Antennas'," Proc. IEEE Veh. Tech. Fall Conference, Sept. 2012.

[9] K. Nagothu, B. Kelley, C. Sekchin, and M. Jamshidi, "Cloud systems architecture for metropolitan area based cognitive radio networks," Proc. IEEE Int. Systems Conference, March 2012.

[10] Harada, H., "Cognitive wireless cloud : A network concept to handle heterogeneous and spectrum sharing type radio access networks," Proc. IEEE Int. Symp. Personal, Indoor and Mobile Radio Communications, Sept. 2009.

[11] Ge Feng et. al., "Cognitive Radio Rides on the Cloud," Proc. IEEE MILCOM, Oct.-Nov. 2010.

[12] I. Gomez, V. Marojevic, and A. Gelonch, "Resource Management for Software-Defined Radio Clouds," IEEE Micro, 2011

[13] (2006) Open Base Station Architecture Initiative, [Online]. Available: at http://ww.obsai.com

[14] (2011) Common Public Radio Interface, [Online]. Available: http://www.cpri.info

[15] (2004) Web Ontology Language (OWL), [Online]. Available: http://www.w3.org/2004/OWL

[16] (2013) RIF Use Cases and Requirements, [Online]. Available: http://www.w3.org/TR/rif-ucr

[17] A. He et al, "A survey of artificial intelligence for cognitive radios," IEEE Trans. Vehicular Tech., vol. 59, no. 4, pp. 1578-1592, May 2010. 